\title{
The Integrated Management Method of Slow Va- rying ISL's for MEO Satellite Network
}

\author{
LI Jing*, YE Gang-qiang*, ZHANG Tian-jiao**, YU Pei-jun* \\ * The State Key Laboratory of Astronautic Dynamics, Xi'an Satellite Control Center, Xi'an China \\ ** Dept of Electronic and Engineering, Xi'an Jiaotong University Xi'an, China \\ carol lee 0727@sina.com
}

\begin{abstract}
In the paper, the constellation composed of MEO (Medium Earth Orbit) satellite is put as a researching scenario, the relative measurement and data transmission task which are finished by slow changing ISLs are put as a researching object, the integrated management of relative measurement and data transmission task inside the satellite network is put as a studying objective. Firstly, the characteristic of relative measurement and data transmission task is analyzed, and the ISLs constrained conditions are researched for both of them. Then, the topology structure of satellite network is designed according to the characteristic of slow changing ISLs and taking account into the specified requirement of measurement and data transmission task. Thirdly, in order to realize integrated management of measurement and data transmission task, the routing strategy is designed according to the topology structure of satellite network. Finally, the researching results will be validated through the example. The technical reference will be provided by the researching results for the fields of space internet application, space based TT\&C (Tracking, Telemetry and Control) and satellite communication in the future.
\end{abstract}

Keywords — satellite network, space based TT\&C, ISL's, routing strategy, topology design

\section{INTRODUCTION}

Satellite communication system appeared in the 1970s. It has been attracting the attention of people since then. Satellite communication was developed tending to networked in order to meet the civil and military requirements after the 1990s. Satellite network technology has been become research hotspot gradually. It is being developed to integrated space and ground network based on IP core recently ${ }^{[1]}$.

Satellite network is a kind of system made of multi-type satellites, which are in intra orbit plane and inter orbit plane. The communication path is provided by satellite node through ISLs (Inter Satellite Links) for the purposes of transmitting signal, providing routing and moving packets ${ }^{[2]}$. Satellite network is a kind of intelligentized system with the characteristic of complex network mode and the topology dynamic change ${ }^{[3-4]}$.

The IRIDIUM system is a worldwide, low earth orbit (LEO) satellite communications system designed to support voice, data, facsimile and paging. The IRIDIUM satellite constellation consists of six orbital planes with eleven satellites in each plane for a total of 66 LEO satellites. The satellites are in a circular orbit at an altitude of approximately $780 \mathrm{~km}$. A lot of researches about system architecture, routing design and management method of IRIDIUM system have been studied by the institutes ${ }^{[5-7]}$. The field experts studied focus on the topology and routing technology to the space internet and ISL simultaneously ${ }^{[8-10]}$.

This paper discusses the integrated management problem of relative measurement and data transmission task in the satellite network. Section II analyses the characteristic of relative measurement and data transmission task and researches the ISLs constrained conditions for both of them. Section III designs the topology structure of satellite network according to the characteristic of slow changing ISLs and taking account into the specified requirement of measurement and transmission task. Section IV designs the routing strategy according to the topology structure of satellite network. The final section provides an example for researching results validation.

\section{TASK DESCRIPTION AND CONSTRAINED CONDITION ANALYSIS}

\section{A. ISL Description}

ISL is one of the important parts to realize various functions in satellite network. It can guarantee a satellite network system operation normally. There are three kinds of ISLs consideration from topology structure, they are intra plane ISL, inter plane ISL and inter layer ISL. The inter layer ISL correspond with multilayered satellite network. ISLs can be divided into slow varying ISLs and dynamic varying ISLs. According to the topology design, the dynamic varying ISLs will be switched regularly and the antenna direction will be changed. Usually the slow varying ISLs will not be switched unless they are demanded.

According to the rule of satellite operation, the slow varying ISLs can be established by only permanent ISLs. Permanent ISLs means the ISL will be connected during whole system period, the three conditions will be met: (1) Geometry visible permanently. (2) Signal reachable. (3) The ISL can be established in the range of elevation and azimuth ${ }^{[11]}$.The 
mathematic mode to figure 1 which permanent ISLs can be established between sat 1 and sat 2 at any moment will be expressed as ${ }^{[12]}$ :

$$
y<\frac{r_{\text {sat } 1}^{2}-R^{2}}{r_{\text {sat } 1}}
$$

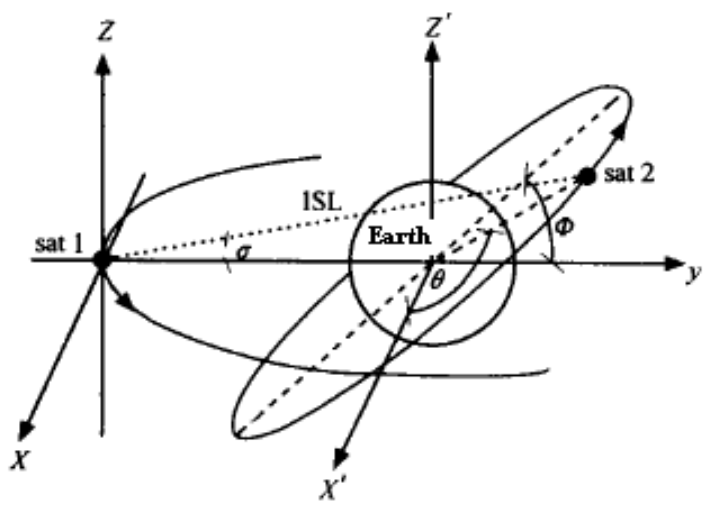

Figure 1. The relative position between two satellites in different orbit plane

Where, $R$ is the Earth radius. $r_{\text {satl }}$ is the orbit radius of sat1.

If the limited time for slow varying ISLs establishment is defined as T, formula (1) will be written as follow:

$y(t)<\frac{r_{\text {sat } 1}^{2}-R^{2}}{r_{\text {sat } 1}} U(t)$

Where,

$$
U(t)= \begin{cases}1 & t \leq T \\ 0 & t>T\end{cases}
$$

\section{B. Task Description for Slow Varying ISLS}

Measurement and data transmission are primary task finished by ISLs. The purpose of measurement is space based $\mathrm{TT} \& \mathrm{C}$ and navigation. The type of data transmission includes real time data and storage data. The slow varying ISL is the optimal choice because it is suitable for telemetry data transmission and relative measurement.

Space based TT\&C mission consists of relative measurement between satellites, telemetry data and tele-command transmission between satellites. Figure 2 shows the slow varying ISLs establishing method in satellite network. The ground TT\&C station will establish the link with only node satellite, and the node satellite can establish the links with multisatellite in the network simultaneously. Every satellite in the network can be used as relay satellite to transmit TT\&C data.

The task set mode of slow varying ISLs will be expressed as follow:

$$
L=\{N S-T S, N S-R S, R S-R S, R S-T S\}
$$

Where, NS-TS is the link between node satellite and target satellite, $N S-R S$ is the link between node satellite and relay satellite, $R S-R S$ is the link between relay satellite and relay satellite, $R S-T S$ is the link between relay satellite and target satellite.

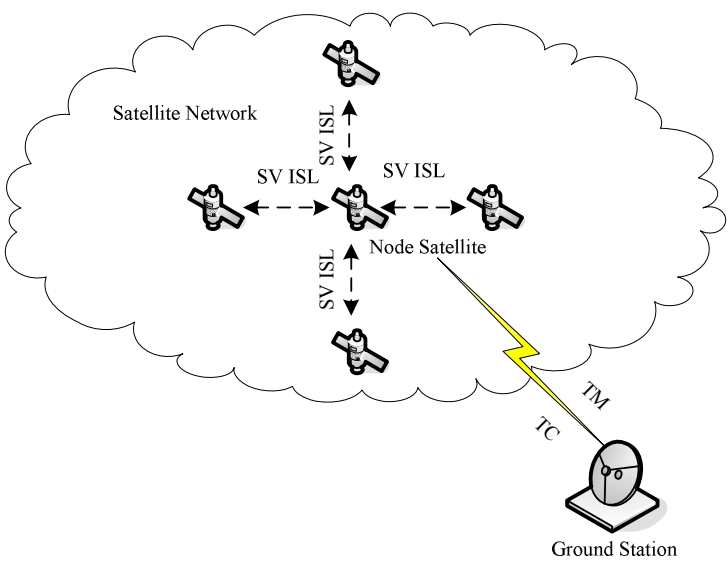

Figure 2. Link establishment sketch of slow varying ISLs

\section{Constrained Condition Analysis of TT\&C Task}

The constrained condition of node satellite TT\&C has been analysed in [13] detailedly. We will describe the constrained condition of slow varying ISLs supported TT\&C following.

\section{1) Geometry Visible Range Between Satellite:}

According to the analysing results of Figure 1 and formula (1), the geometry visible condition between satellites ( the range from sat1 to sat2) can be expressed as follow :

$$
R_{\text {sat }}<\frac{r_{\text {sat } 1}^{2}-R^{2}}{r_{\text {sat } 1}}
$$

\section{2) Maximum Direction Angle Between Satellite:}

According to the analysing results of Figure 1, the maximum direction angle between satellites ( the angle from sat1 direct to sat2 ) can be expressed as follow:

$$
\begin{aligned}
& \text { max_ } \sigma_{\text {sat } 1} \leq \arcsin \left[\frac{r_{\text {sat } 2 \sin \phi}}{\sqrt{r_{\text {sat } 1}^{2}+r_{\text {sat } 2}^{2}-2 r_{\text {sat } 1} r_{\text {sat } 2} \cos \phi}}\right] \\
& \text { max_} \sigma_{\text {sat } 2} \leq \arcsin \left[\frac{r_{\text {sat } 1} \sin \phi}{\sqrt{r_{\text {sat } 1}^{2}+r_{\text {sat } 2}^{2}-2 r_{\text {sat } 1} r_{\text {sat } 2} \cos \phi}}\right]
\end{aligned}
$$

Where, $\phi$ is the angle between two orbit plane.

\section{3) Signal Reachable Range Between Satellite:}

Inside Figure 1, assumption: the equivalent isotropic radiated power of sat1 is $E I R P_{\text {satl }}$, the antenna gain of sat2 is $G_{\text {sat } 2}$

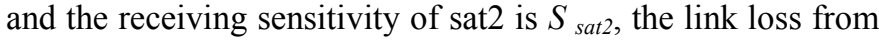
antenna to receiver input port is $L_{\text {sat } 2}$, the space loss from sat1 to sat2 is $L_{s p}$, the link frequency is $f$. The signal reachable range can be expressed as follow:

$R_{\text {signal }}=10^{\frac{1}{20}\left[E I R P_{\text {sat } 1}-20 \lg f(M H z)+G_{\text {sat } 2}-L_{\text {sat } 2}-S_{\text {sat } 2}-32.45\right]}(\mathrm{km})$

\section{4) Basic Condition of Link Establishment:}

Consideration the analysing results of 1) - 3), the basic condition of link establishment is expressed as: 


$$
R_{L}=R_{\text {sat }} \bigcap \text { max_ } \sigma_{\text {sat }} \bigcap R_{\text {signal }}
$$

Where, $R_{\text {sat }}$ is the link member in network satisfied formula (4), $\max \sigma_{\text {sat }}$ is the link member in network satisfied formula (5) and (6), $R_{\text {signal }}$ is the link member in network satisfied formula (7).

\section{5) Priority of Link Establishment:}

According to the description of formula (3), the priority of link establishment from high to low is expressed as:

$L_{p r i}=\{N S-T S \rightarrow R S-T S \rightarrow N S-R S \rightarrow R S-R S\}$

\section{6) Link Establishment Condition Simultaneous:}

In the network, the number of ISLs of mono-satellite is depended to number of channels onboard. If the number of channels is expressed as $N U M_{\text {sat }}$, the link establishment condition simultaneous can be expressed as:

$$
N U M_{\text {ISL }} \leq N U M_{\text {sat }}
$$

\section{III.TOPOLOGY DESIGN AND ROUTING STRATEGY}

\section{A. Structure of MEO Satellite Network}

In Walker constellation, there are permanent ISLs between the satellites which are close enough on intra orbit plane or on inter orbit plane. If the link specification is good enough, the permanent ISLs will also be established between the satellites which are apart on intra orbit plane because the orbit altitude is higher for MEO satellite ${ }^{[14][15]}$. Figure 3 shows a typical structure of MEO constellation network. Here, the permanent ISLs are expressed as solid lines.

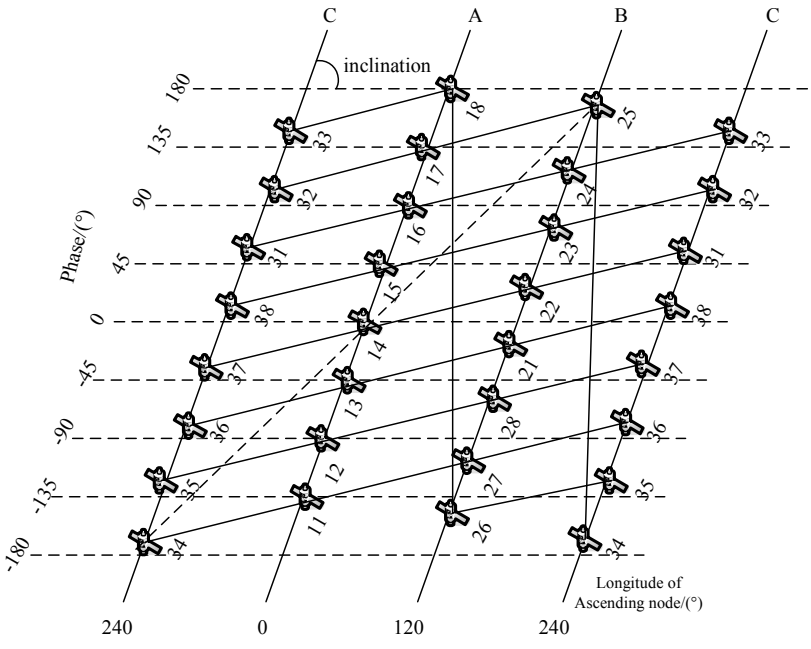

Figure 3. Structure sketch of MEO satellite network

\section{B. Topology Structure of Slow Varying ISLs}

ISLs topology structure is established on the base of satellite network structure. It is different from the previous research, the topology structure of slow varying ISLs we discussed in this paper with the following characteristics ${ }^{[16]}$.

- Any mono-satellite in the network is able to establish multi-ISL simultaneously.

- ISL in the network is peer link.
- The whole constellation will be managed by any satellite in the network.

- In order to insure the measurement effective, the link will be established on both intra orbit plane and inter orbit plane ${ }^{[17]}$.

Assume: the MEO satellite network consists of $\mathrm{P}$ orbital planes with $\mathrm{K} / \mathrm{P}$ satellites in each plane for a total of $\mathrm{K}$ satellites. There are $\mathrm{M}$ permanent ISLs, and the information from $\mathrm{N}$ links can be received and transmitted simultaneously by each satellite. Here, $\mathrm{M} \geq \mathrm{N}$.

The heuristic graph planning method will be applied to design the topology structure because of the better stability of slow varying ISLs. The condition in formula (8) will be satisfied by the elements in heuristic information set. The problem of ISL topology design can be expressed with quaternary group.

$\Pi=\langle S A T, I, O, G\rangle$

Where, $S A T$ is objectives set, it will be expressed as:

$$
S A T=\left\langle s a t_{11}, \cdots s a t_{1 K / P} ; \cdots s a t_{P 1}, \cdots s a t_{P K / P}\right\rangle
$$

$I$ is initial conditions set, it will be expressed as:

$I=\langle I S L 1, I S L 2, \cdots I S L N\rangle$

$O$ is operators set, it will be expressed as:

$O=\langle$ connect with orbit plane $1, \cdots$ connect with orbit plane $P\rangle$

$\mathrm{G}$ is goals set, it will be expressed as:

$$
G=\left\langle\text { sat }_{i j} \text { connect with } S A T\right\rangle
$$

The meaning of formula (15) is that the whole constellation can be managed by satellite and ground link with any satellite as a node. If the operation sequence is $\mathrm{o}_{1}, \mathrm{o}_{2}, \ldots \mathrm{o}_{\mathrm{n}}$, then

$$
\operatorname{app}_{o_{n}}\left(\operatorname{app}_{o_{n}-1}\left(\cdots \operatorname{app}_{o_{1}}(I) \cdots\right)\right) \mid=G
$$

\section{Design of Routing Strategy}

Routing technology is the key point in the field of network communication. In the early satellite communication system, GEO satellite is used as relay satellite, the data was be transmitted between two places by bent-pipe transponder. The mode of this kind of data transmission is fixed, and no routing. In the constellation network consisted of multi nonsynchronization satellites, the optimal routing will be selected between the source node satellite to the target node satellite according to the given cost function ${ }^{[18]}$.

Here, we use graph $\operatorname{GRP}(S A T, \operatorname{LINK}, W)$ to express MEO satellite network:

$S A T$ is node satellites set in the network which is expressed as formula (12).

$$
\begin{aligned}
& \text { LINK }=\left\langle\operatorname{link}_{1}, \operatorname{link}_{2} \cdots, \operatorname{link}_{j}\right\rangle \text { is ISL set. } \\
& W_{i}\left(\operatorname{link}, t_{j}\right)=\left\langle w_{1}\left(\operatorname{link}, t_{1}\right), w_{2}\left(\operatorname{link}, t_{2}\right), \cdots w_{k}\left(\operatorname{link}, t_{j}\right)\right\rangle \text { is link }
\end{aligned}
$$

metric set at the moment of $t_{j}$, we have $1<j<k$, and $k$ is metric parameter.

The main purpose of routing design is to research the cost metric method of satellite routing from source node satellite to target node satellite. 


\section{1) Selecting Strategy of Node Satellite:}

Any satellite can be used as node satellite to establish the satellite and ground link in MEO constellation network because of peer link characteristic. Therefore, the node satellite will be selected as the following step.

Step one: task analysis.

The ground TT\&C node will be selected according to the $\mathrm{TT} \& \mathrm{C}$ and communication task demands. There are over two ground TT\&C station are needed to determine satellite precision orbit, and the two ground station are located as far as possible. There is only one ground TT\&C station are needed to transmit telemetry and tele-command data.

\section{Step two: visible analysis.}

The visible matrix will be calculated according to the selection result of ground TT\&C station. It will be expressed as follow:

$\mathrm{L}=\left[\begin{array}{cccc}L\left(\text { station }- \text { sat }_{1}, t_{1}\right) & L\left(\text { station }-s a t_{2}, t_{1}\right) & \cdots & L\left(\text { station }-s a t_{K}, t_{1}\right) \\ L\left(\text { station }-s a t_{1}, t_{2}\right) & L\left(\text { station }-s a t_{2}, t_{2}\right) & \cdots & L\left(\text { station }-s a t_{K}, t_{2}\right) \\ \vdots & \vdots & \vdots & \vdots \\ L\left({\left.\text { station }-s a t_{1}, t_{i}\right)}\right) & L\left(\text { station }-s a t_{2}, t_{i}\right) & \cdots & L\left(\text { station }-s a t_{K}, t_{i}\right)\end{array}\right]$

Where, " 1 " is used to express link connection, " 0 " is used to express no link.

Step three: node satellite selecting.

The priority sequence is established according to duration of satellite and ground link.

\section{2) Optimal Routing Strategy:}

\section{Step one: path calculation.}

The possible path will be selected with the suitable algorithm according to ISL topology, source node satellite and target node satellite, consideration of link establishment constrained condition. The set can be expressed as follow:

$$
P A T H=\langle N S-T S, N S-R S, R S-R S, R S-T S\rangle
$$

\section{Step two: path optimization.}

The path will be sorted according to the priority described by formula (9). The selected rule is time delay minimum.

\section{3) Routing Maintenance Strategy:}

The routing is needed to maintenance or reconfiguration when the satellite or ISL is disconnected or invalidation. If the satellite is invalidation, another optimal node will be selected from node satellite sequence, and the optimal path will be selected. If the ISL is invalidation, another optimal path will be selected from optimal routing sequence.

It is a sub-course above which is an example of managing satellite network with ground station. After the selected node satellite invisible, the next course is coming. We can choose the same station as a ground $\mathrm{TT} \& \mathrm{C}$ node or another one.

\section{IV.DESIGN ANALYSIS}

\section{A. Scenario Description}

The scenario is divided into space section and ground section. The space section is a Walker $24 / 3 / 1$ constellation consisted of 24 MEO satellites, the orbit height is over $20000 \mathrm{~km}$ and orbit inclination is $55^{\circ}$. Each satellite is able to establish
3ISLs at the same time. The ground section is consisted of 4 TT\&C stations located in northeast, northwest, south and middle of China, name as Mid_Station, NE_Station, NW_Station and Sth_Station respectively.

\section{B. Description of task demands}

Generally, the task can be described as: TT\&C and communication management for the whole constellation will be operated incessantly with as less as possible ground TT\&C resource through establishing satellite network. There are three parts involved as follow:

- The designed topology will be suitable for satellite orbit determination.

- The optimal routing is easy to manage TT\&C data transmission.

- The network routing can be maintained and reconfigured.

\section{Topology Analysis}

Figure 3 shows the topology of space network. The following principles will be followed when designing ISLs topology.

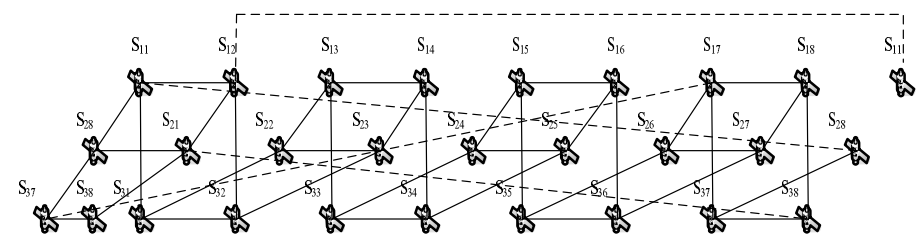

Figure 4. Topology structure of slow varying ISL

- The ISLs will be established with the nearest satellite on both intra orbit plane and two inter orbit plane.

- The ISLs topology structure will be not changed without new input driving.

- The whole constellation will be managed by ground TT\&C station with only one node satellite.

According to the above principles, the slow varying ISLs topology is designed as Figure 4.

\section{Routing Analysis}

According to the routing optimization strategy showed in chapter three of this paper, the four links matrix from satellite to ground are calculated, and the node satellite sets sorted with visible duration are designed.

The set related with Mid Station is as follow:

$N S_{1}=\left\langle S_{21}, S_{37}, S_{22}, S_{38}, S_{23}, S_{31}, S_{13}, S_{14}, S_{15}\right\rangle$.

The set related with NW_Station is as follow:

$N S_{2}=\left\langle S_{22}, S_{38}, S_{23}, S_{31}, S_{13}, S_{24}, S_{32}, S_{14}, S_{15}\right\rangle$.

The set related with NW_Station is as follow:

$N S_{3}=\left\langle S_{21}, S_{22}, S_{38}, S_{13}, S_{14}, S_{15}, S_{31}, S_{23}\right\rangle$,.

The set related with $S$ th Station is as follow:

$N S_{4}=\left\langle S_{14}, S_{21}, S_{15}, S_{37}, \bar{S}_{22}, S_{38}, S_{23}, S_{31}, S_{24}, S_{16}\right\rangle$. 
Assume: we will choose one ground TT\&C station to receive telemetry data of target node satellite $\mathrm{S}_{11}$. The following step will be followed.

Step one: To determine set $N S_{i}$ whether includes element $\mathrm{S}_{11}$ or not. If it includes $\mathrm{S}_{11}$, the telemetry data will be transmitted by satellite to ground link directly. But, the $\mathrm{S}_{11}$ is invisible for four ground TT\&C station.

Step two: The ISL length from possible origin node satellites to end node satellite according to the $N S_{i}$ set and ISL topology.

Step three: After consideration ground resource and data throughput, we will choose origin node to establish the link between satellite and ground.

After analysis the elements in the set $N S_{i}$, we can know: twelve satellites are possessed of establishment ISLs condition. If we select the shortest path from each origin node satellites to end node satellite, there will be twelve usable paths. They are:

$$
R T=\left\langle R T_{1}, R T_{2}, R T_{3}, R T_{4}, R T_{5}, \cdots, R T_{12}\right\rangle
$$

Where,

$$
\begin{aligned}
& R T_{1}=\left\{S_{13} \rightarrow S_{22} \rightarrow S_{31} \rightarrow S_{11}\right\} \\
& R T_{2}=\left\{S_{14} \rightarrow S_{23} \rightarrow S_{32} \rightarrow S_{12} \rightarrow S_{11}\right\} \\
& R T_{3}=\left\{S_{15} \rightarrow S_{24} \rightarrow S_{33} \rightarrow S_{13} \rightarrow S_{22} \rightarrow S_{31} \rightarrow S_{11}\right\} \\
& R T_{4}=\left\{S_{16} \rightarrow S_{36} \rightarrow S_{27} \rightarrow S_{18} \rightarrow S_{38} \rightarrow S_{21} \rightarrow S_{12} \rightarrow S_{11}\right\} \\
& R T_{5}=\left\{S_{21} \rightarrow S_{28} \rightarrow S_{11}\right\} \\
& R T_{6}=\left\{S_{22} \rightarrow S_{31} \rightarrow S_{11}\right\} \\
& R T_{7}=\left\{S_{23} \rightarrow S_{22} \rightarrow S_{31} \rightarrow S_{11}\right\} \\
& R T_{8}=\left\{S_{24} \rightarrow S_{33} \rightarrow S_{13} \rightarrow S_{31} \rightarrow S_{11}\right\} \\
& R T_{9}=\left\{S_{31} \rightarrow S_{11}\right\} \\
& R T_{10}=\left\{S_{32} \rightarrow S_{12} \rightarrow S_{11}\right\} \\
& R T_{11}=\left\{S_{37} \rightarrow S_{28} \rightarrow S_{11}\right\} \\
& R T_{12}=\left\{S_{38} \rightarrow S_{21} \rightarrow S_{12} \rightarrow S_{11}\right\}
\end{aligned}
$$

After analysis the twelve routings, the optimization routing sequence will be obtained as:

$$
R T_{\text {opt }}=\left\langle R T_{9}, R T_{10}, R T_{11}, R T_{5}, R T_{6}, R T_{12}, R T_{7}, R T_{1}, R T_{8}, R T_{2}, R T_{3}, R T_{4}\right\rangle
$$

The results will be obtained from set $R T_{\text {opt }}$ and $N S_{i}$ : if we select $\mathrm{S}_{37}$ as an origin node satellite, the shortest path and the most of ground resource will be got.

\section{CONCLUSION}

The researching aim of this paper is to realize space based TT\&C and communication in MEO satellite network. The ISLs topology and optimal routing strategy are studied to slow varying ISLs. The method of topology design for MEO satel- lite network is proposed, which take account into the specified requirement of measurement and transmission task. The optimal routing selection strategy based on slow varying ISLs topology is designed. Finally, the scenario is established which consisted of $24 \mathrm{MEO}$ satellites and four ground TT\&C stations, and it is analysed as a example. The analysing results show: the integrated management problem of measurement and data transmission can be solved well by the methods and strategies proposed this paper. The technical reference will be provided by the researching results for the fields of space internet application, space based TT\&C (Tracking, Telemetry and Control) and satellite communication in the future.

\section{REFERENCES}

[1] H. D. Clausen, H. Linder, and B. Collini-Nocker, "Internet over Direct Broadcast Satellites," IEEE Communications Magazine, vol.37, pp. 146-151, Jun. 1999.

[2] W. WERNER, A. JAHN, and E. LUTZ, "Analysis of System Parameters for LEO / ICO Satellite Communication Networks," IEEE Journal on Selected Areas in Communications, vol. 13, pp. 371-381, Feb. 1995.

[3] I.F.AKYILDIZ, and S. JEONG, "Satellite ATM Networks: A Survey," IEEE Communications Magazine, vol.35, pp. 30-44, Jul. 1997.

[4] P. B. Jensen and D. Moshenberg, "The AT\&T Satellite Network Control System," IEEE Journal on Selected Areas in Communications, vol. 10, pp. 1094-1102, Jun. 1992.

[5] C. E. Fossa, R. A. Raines, G. H. Gunsch,and M. A. Temple, “An Overview of the IRIDIUM Low Earth Orbit (LEO) Satellite System, "in IEEE Aerospace and Electronics Conference, 1998, pp. 152-159.

[6] K. M. Peterson, "The Iridium Low Earth Orbit Communications System,” in IEEE Sarnoff Symposium, 1994, pp. 13-19.

[7] S. R. Pratt, "Performance Analysis of Dynamic Routing Algorithms in an IRIDIUM-Like Low Earth Orbit Satellite System," Rep. AFIT/GCE/ENG/99M-04, 1999.

[8] Aida Nathalie Urquizo Medina,and Gao Qiang, "QoS Routing for LEO Satellite Networks," Lecture Notes in Computer Science, vol. 7719, pp. 495-512, Jan. 2013.

[9] Y.HU, and V. O. K. LI, "Satellite-based Internet: A Tutorial," IEEE Communications Magazine, vol.39, pp. 154-162, March 2001.

[10] Zihe Gao, Qing Guo and Zhen yu Na, "Novel Optimized Routing Algorithm for LEO Satellite IP Networks," Journal of Systems Engineering and Electronic, vol. 22, pp. 917-925, Jun. 2011.

[11] Jing LI, Hong-jun HU, Pei-jun YU and Jun ZHU, "Analysis of Requirement and Constrained Model of Inter-Satellite-Link TT\&C Scheduling Problem on Navigation Constellation," Artificial Intelligence and Computational Intelligence, vol. 7530, pp. 160-169, Oct. 2012.

[12] WANG Zhen-yong, WANG Ping, GU Xue-mai and GUO Qing, "Research on Design of Permanent Inter-Satellite-1inks in Satellite Networks," Journal on Communications, vol. 27, pp. 129-133, Aug. 2006.

[13] LI Jing, YU Pei-jun and LIU Jian-ping, "The TT\&C Resource Scheduling Method Based on Multi-satellite, "in Proc. IEEE ICIS2010, pp. 431-435, 2010.

[14] LI Zhendong, HE Shanbao, LIU Chonghua and WANG Haihong, "A Topology Design Method of Navigation Satellite Constellation Inter2satellite Links," Spacecraft Engineering, vol. 20, pp. 32-37, Mar. 2011.

[15] Lin Binjie, "Research on the Topology and Routing Protocol of Multilayered Satellite Network," M. Eng. thesis, University of Science and Technology of China, Jan. 2010.

[16] P.Takats, "A New Paradigm: Geostationary Satellite Clusters with Inter-satellite Links," in 21st AIAA International Communications Satellite Systems Conference, pp. 1-8, 2003.

[17] WEN Yuan-lan, LIU Qi-xu, and ZHU Jun, "The Effect of TT\&C Deployment on the Regional Satellite Navigation System," Journal of National University of Defense Technology, vol. 29, pp. 1-6, Jan. 2007.

[18] L. Franek, and G. Maral, "Routing in Networks of Inter Satellite Links," IEEE Transactions on Aero Space and Electronic Systems, vol. 38, pp. 902-917, Mar. 2002. 